\title{
A novel approach to the solution of a steady state biofilter model
}

\author{
Mubarak Salih ${ }^{1}$, Zarook Shareefdeen ${ }^{1 \dagger}$, Suheil Khouri ${ }^{2}$ \\ ${ }^{1}$ Department of Chemical Engineering, American University of Sharjah, 26666, Sharjah, United Arab Emirates \\ ${ }^{2}$ Department of Mathematics and Statistics, American University of Sharjah, 26666, Sharjah, United Arab Emirates
}

\begin{abstract}
Designing of air pollution control equipment such as biofilters, also known as gas phase biological reactors, for elimination of toxic volatile organic compounds (VOCs) from air streams require accurate modeling and model solutions. In this work, a novel approach is employed to solve a biofilter model and for performance predictions. The approach is based on expressing the particular solution of the governing equations as an integral containing Green's function. The integral is then tuned to develop a suitable operator in which Krasnoselskii-Mann's fixed point iteration method is applied. In this work, this technique was utilized to model the biological degradation of methanol and alpha-pinene. The results obtained by the proposed technique were compared against the experimental data adopted from literature and the originally published numerical solution. The comparison showed that the approach is highly accurate and converges fast with a uniformly distributed residual error over the entire domain. Furthermore, the method was used to perform a sensitivity analysis on the model equations to evaluate the performance of the biofilter under various conditions. To the authors' knowledge, this is the first time Mann's Green's embedded method (MGEM) is applied in the solution of biofilter models.
\end{abstract}

Keywords: Biofiltration, Green's function, Krasnoselskii-Mann's fixed point iteration, Mathematical model, Volatile organic compounds (VOCs)

\section{Introduction}

Primary air pollutants such as volatile organic compounds (VOCs) and odorous compounds are responsible for several ecological problems such as ozone layer depletion, worldwide temperature changes, global warming and worsening of air quality [1]. Consequently, the demand for air pollution control to eliminate the emissions of these pollutants has increased. A new energy management system is explored to benefit from the global warming effects due to air pollutant release [2]. In addition to that, air pollution regulations enforced by environmental protection agencies have driven industries to use efficient air treatment processes in order to meet air quality standards [3]. Among the available air treatment processes, biological treatment methods such as biofiltration received tremendous popularity and interest [4]. This is attributed to the fact that, classical pollution control technologies like adsorption, absorption and incineration are becoming more expensive because of stringent legislations on waste management and pollution control [5]. A detailed comparison of several classical technologies of VOC removal against biofiltration is discussed by Malakar et al. [6].

This is an Open Access article distributed under the terms of the Creative Commons Attribution Non-Commercial License (http://creativecommons.org/licenses/by-nc/3.0/) which permits unrestricted non-commercial use, distribution, and reproduction in any medium, provided the original work is properly cited.

Copyright (C) 2020 Korean Society of Environmental Engineers
In biofilters (BFs), polluted air passes through a pretreatment unit in which particulate matters are removed and the air is humidified [1]. The humid air stream then enters a bioreactor packed with porous media on which microbial consortium like bacteria, yeast or fungi is attached. A simple schematic of a biofilter is shown in Fig. 1. The pollutants in the air are transferred to the attached microorganisms, known as biofilm where biological oxidation takes place. The biological oxidation of VOCs produces carbon dioxide, water, biomass and metabolic by-products. Biofiltration is attractive due to many reasons including high removal efficiency (RE) in treating large volumes of air containing low concentrations of pollutants; no secondary waste stream is produced in this process and it is an environmentally friendly process. Despite many attractive features associated with $\mathrm{BF}$, they also have few disadvantages that include less suitability for high concentrations of pollutants, difficulty in $\mathrm{pH}$ and moisture control and deterioration of packing media over a long time use [4].

The operation of BFs is affected by several factors such as biofilm thickness, oxygen level, and pressure drop, type of packing material used, $\mathrm{pH}$ and nutrients [7-12]. Understanding the influence of these
Received October 4, 2019 Accepted October 17, 2019
${ }^{\dagger}$ Corresponding author
Email: zshareefdeen@aus.edu
Tel: +971-6-515-2988 Fax: +971-6-515-2979
ORCID: 0000-0001-5051-2504 


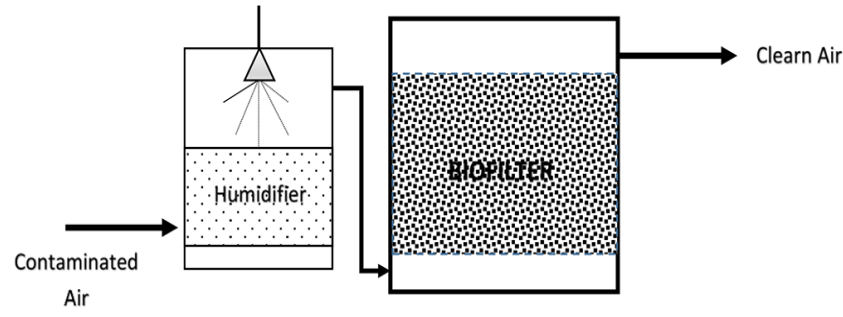

Fig. 1. A simple schematic diagram of a biofilter.

factors on the biofiltration process has been brought out through mathematical models. The biofilter mathematical models help engineers and researchers design industrial-scale biofiltration units, guide experimentation and predict the system behavior under different conditions. A number of biofilter models ranging from very simple to more complex models have been developed [13-26]. These models differ essentially in the following aspects which are the gas flow pattern, the mass exchange between the gas phase and the biofilm phase, the type of microbial growth kinetics, the nature of pollutants being treated and the role of the packing material used [27].

The BF model equations are derived by relating microbial growth kinetics to bioreactor design equations. The bioreactor design equations are derived by setting up a general mass balance within the biofilm phase and along the BF height. The solution of these models requires a simultaneous evaluation of the pollutants' concentrations in the gas phase and in the biofilm phase; however, the mathematical formulation of BFs with non-linear kinetics have no analytical closed-form solutions and the numerical algorithms are typically used to obtain approximate solutions.

The objective of this work is to apply the iterative scheme of Green's function Krasnoselskii-Mann's fixed point iteration [28] which is also recognized as Mann's Green's embedded method (MGEM) to find an approximated solution for a BF model developed by Mohseni and Allen [19]. The Mann's strategy overcomes deficiencies of other iterative and numerical schemes namely the deterioration of the numerical results and errors as the domain increases. An important characteristic of the present method is that it ensures uniform convergence since the construction of Green's function takes into account both endpoints of the boundary-value problem's domain [29]. In this work, this novel technique is utilized to model biofiltration of methanol and alpha-pinene, which are key hazardous air pollutants emitted from the pulp and paper industry. Methanol is listed as one of the 189 hazardous air pollutants (HAPs) listed in Title III of the 1990 Clean Air Act [19]. The proposed iterative scheme was also used to perform a sensitivity analysis on the model equations in order to quantify the effects of important parameters on the biofiltration process.

\subsection{Mathematical Model}

The model of Mohseni and Allen [19] was developed to investigate the treatability of methanol $(\mathrm{MeOH})$ and alpha-pinene as single pollutants or in a mixture using a BF packed with compost and wood chip. The detailed assumptions used to derive the $\mathrm{BF}$ model and the model equations are presented in sections 2.1 and 2.2 .

\subsection{Model assumptions}

In Mohseni and Allen model [19], the flow of air throughout the $\mathrm{BF}$ column is adequately taken as plug flow neglecting the radial concentration gradient and the effect of axial dispersion. At the air-biofilm interface, the biofilm concentrations of $\mathrm{MeOH}$ and alpha-pinene are assumed to be at equilibrium with the concentrations in the air, and the concentrations are related by Henry's law. The thickness of the biofilm is considered small relative to the diameter of the packing material; hence they used planar geometry. With regard to the mass transfer, molecular diffusion is presumed to be responsible for transporting $\mathrm{MeOH}$ and alpha-pinene into the biofilm. In the biofilm, the biodegradation is described by the microbial growth kinetics such that Monod kinetics is used for $\mathrm{MeOH}$ and Monod kinetics with noncompetitive inhibition is used for alpha-pinene.

\subsection{Model Equations}

The ordinary differential equations (ODEs) describing the concentrations of $\mathrm{MeOH}$ and alpha-pinene in the biofilm are given by (1) and (2); the conditions at the boundaries are specified by (3) and (4). The notations $D, Y, X_{V}, \delta, \mu_{\max }, K_{S}, K_{\mathrm{I}}, \alpha, m$ and $x$ symbolize the diffusion coefficient in the biofilm, the biomass yield coefficient, the dry cell density of the biofilm, the biofilm thickness, the maximum specific growth rate, the half-saturation constant, the inhibition constant for alpha-pinene in the presence of $\mathrm{MeOH}$, the coefficient for the effect of $\mathrm{MeOH}$ on alpha-pinene biodegradation, the air-biofilm partition coefficient and the distance in the biofilm, respectively. While $S, S_{i}$ and $C$ denote the concentration in the biofilm, the concentration at the air-biofilm interface and the concentration in the air stream, respectively. The subscripts $m$ and $P$ represent $\mathrm{MeOH}$ and alpha-pinene, respectively.

Biofilm phase model equations:

$$
\begin{gathered}
D_{m} \frac{d^{2} S_{m}}{d x^{2}}=\frac{X_{v}}{Y_{m}} \frac{\mu_{\max (m)} S_{m}}{K_{S(m)}+S_{m}} \\
D_{p} \frac{d^{2} S_{p}}{d x^{2}}=\alpha \frac{X_{v}}{Y_{p}} \frac{\mu_{\max (p)} S_{p}}{K_{S(p)}+S_{p}}
\end{gathered}
$$

where,

$$
\alpha=\frac{1}{1+\left(\frac{C_{m}}{K_{I}}\right)^{2}}
$$

The associated boundary conditions are:

$$
\begin{gathered}
S_{m}=\frac{C_{m}}{m_{m}} \text { and } S_{p}=\frac{C_{p}}{m_{p}} \text { at } x=0 \\
\frac{d S_{m}}{d x}=\frac{d S_{p}}{d x}=0 \quad \text { at } \quad x=\delta
\end{gathered}
$$


The ODEs describing the concentrations of $\mathrm{MeOH}$ and alpha-pinene in the gas phase have the forms of (5) and (6); the entrance conditions are shown by (7) and (8). In these equations $U_{g}$, $A_{s}, h$ and $C_{i}$ stand for the air superficial velocity, the biofilm surface area per unit volume of the $\mathrm{BF}$, the position along the $\mathrm{BF}$ height and the concentration in the inlet air stream, respectively.

Gas phase model equations:

$$
\begin{aligned}
& U_{g} \frac{d C_{m}}{d h}=A_{S} D_{m}\left(\frac{d S_{m}}{d x}\right)_{x=0} \\
& U_{g} \frac{d C_{p}}{d h}=A_{S} D_{p}\left(\frac{d S_{p}}{d x}\right)_{x=0}
\end{aligned}
$$

The associated entrance conditions are:

$$
\begin{aligned}
& C_{m}=C_{i(m)} \text { at } h=0 \\
& C_{p}=C_{i(p)} \text { at } h=0
\end{aligned}
$$

The model equations, the corresponding boundary and entrance conditions are made dimensionless by defining the following dimensionless parameters:

$$
\begin{gathered}
S_{m}^{*}=\frac{S_{m}}{S_{i(m)}}, \quad S_{p}^{*}=\frac{S_{p}}{S_{i(p)}}, \quad C_{m}^{*}=\frac{C_{m}}{C_{i(m)}}, C_{p}^{*}=\frac{C_{p}}{C_{i(p)}}, \sigma=\frac{x}{\delta}, \\
\xi=\frac{h}{H}, \phi_{1}=\frac{X_{v} \mu_{\max (m)} \delta^{2}}{Y_{m} D_{m} K_{S(m)}}, \phi_{2}=\frac{X_{v} \mu_{\max (p)} \delta^{2}}{Y_{p} D_{p} K_{S(p)}}, \beta_{1}=\frac{C_{i(m)}}{m_{m} K_{S(m)}}, \\
\beta_{2}=\frac{C_{i(p)}}{m_{p} K_{S(p)}}, \quad \kappa_{1}=\frac{H D_{m} A_{S}}{m_{m} U_{g} \delta}, \quad \kappa_{2}=\frac{H D_{p} A_{S}}{m_{p} U_{g} \delta} .
\end{gathered}
$$

Using the dimensionless parameters, the model equations and the corresponding boundary and entrance conditions become:

Dimensionless biofilm phase model equations:

$$
\begin{gathered}
\frac{d^{2} S_{m}^{*}}{d \sigma^{2}}=\phi_{1} \frac{S_{m}^{*}}{1+\beta_{1} C_{m}^{*} S_{m}^{*}} \\
\frac{d^{2} S_{p}^{*}}{d \sigma^{2}}=\alpha \phi_{2} \frac{S_{p}^{*}}{1+\beta_{2} C_{p}^{*} S_{p}^{*}}
\end{gathered}
$$

where:

$$
\alpha=\frac{1}{1+\left(\frac{C_{m}}{K_{I}}\right)^{2}} .
$$

The associated dimensionless boundary conditions are:

$$
S_{m}^{*}=S_{p}^{*}=1 \text { at } \sigma=0
$$

$$
\frac{d S_{m}^{*}}{d \sigma}=\frac{d S_{p}^{*}}{d \sigma}=0 \quad \text { at } \quad \sigma=1
$$

Dimensionless gas phase model equations:

$$
\frac{d C_{m}^{*}}{d \xi}=\kappa_{1} C_{m}^{*}\left(\frac{d S_{m}^{*}}{d \sigma}\right)_{\sigma=0}
$$

$$
\frac{d C_{p}^{*}}{d \xi}=\kappa_{2} C_{p}^{*}\left(\frac{d S_{p}^{*}}{d \sigma}\right)_{\sigma=0}
$$

The associated dimensionless entrance conditions are:

$$
C_{m}^{*}=1 \text { at } \xi=0
$$

$$
C_{p}^{*}=1 \quad \text { at } \quad \xi=0
$$

\section{Presentation of the Iterative Scheme}

The Mann's Green's embedded method (MGEM) has been developed by M. Abushammala et al. [28] to find approximated solutions for linear and nonlinear third order ODEs that arise in physical applications. The advantages of using this iterative scheme include fast convergence to the solution; and it gives highly accurate results without the need for spatial discretization or any assumptions that might change the physical representation of the model.

\subsection{The Iterative Scheme for the Solution of First Order ODEs}

Consider the following generalized first order ODE where $L$ is a linear differential operator representing the first order derivative and $f(x)$ is the inhomogeneous term.

$$
L[y] \equiv y^{\prime}(x)+p(x) y(x)=f(x)
$$

subject to the following initial condition:

$$
B[y] \equiv y(a)=A_{1}
$$

The general solution to (17) is given as:

$$
y=y_{h}+y_{p}
$$

In (19), $y_{h}$ is the homogeneous solution which satisfies $L[y]=0$ and the initial condition is given by (18). While $y_{p}$ is the particular solution which satisfies $L[y]=f(x)$ and the following initial condition $B[y]=0$.

The particular solution to (17) is represented as:

$$
y_{p}=\int_{a}^{\infty} G(x, s) f(s) d s
$$


The Green's function $G(x, s)$ is defined as:

$$
L[G(x, s)]=\delta(x-s)
$$

subject to:

$$
B[G(x, s)]=0
$$

For $x \neq s$, the Green's function is a homogeneous solution to (17). However, when $x=s$ the Green's function has singular behavior. Therefore, it comprises of two identical solutions for each side of the Dirac delta function given as:

$$
G(x, s)= \begin{cases}e_{1} \exp \left(-\int_{s}^{x} p(x) d x\right) & a<x<s \\ e_{2} \exp \left(-\int_{s}^{x} p(x) d x\right) & x>s\end{cases}
$$

where $e_{1}$ and $e_{2}$ are constants; these constants are to be found using the following properties:

$G(x, s)$ satisfies the homogeneous initial condition:

$$
B[G(x, s)]=0
$$

Jump discontinuity of $G(x, s)$ at $x=s$ :

$$
e_{2} \exp \left(-\int_{s}^{x} p(x) d x\right)-e_{1} \exp \left(-\int_{s}^{x} p(x) d x\right)=1
$$

For the implementation of the MGEM, we first define the following linear integral operator:

$$
K[y]=y_{h}+\int_{a}^{\infty} G(x, s)\left[y^{\prime}(s)+p(s) y(s)\right] d s
$$

Now adding and subtracting $f(x)$ from (26), we get:

$$
K[y]=y_{h}+\int_{a}^{\infty} G(x, s)\left[y^{\prime}(s)+p(s) y(s)-f(s)\right] d s+\int_{a}^{\infty} G(x, s) f(s) d s
$$

Using (19) and (20) in (27), we obtain:

$$
K[y]=y_{h}+\int_{a}^{\infty} G(x, s)\left[y^{\prime}(s)+p(s) y(s)-f(s)\right] d s+y-y_{h}
$$

Therefore, from (28), we find:

$$
K[y]=y+\int_{a}^{\infty} G(x, s)\left[y^{\prime}(s)+p(s) y(s)-f(s)\right] d s
$$

Mann's fixed point iterative scheme is known as:

$$
y_{n+1}=\left(1-\alpha_{n}\right) y_{n}+\alpha_{n} K[y] \quad \text { for } \quad n \geq 0
$$

Applying (30) to (29) yields:

$$
y_{n+1}=y_{n}+\alpha_{n} \int_{a}^{\infty} G(x, s)\left[y_{n}^{\prime}(s)+p(s) y_{n}(s)-f(s)\right] d s
$$

For $n=0$, the starting function $y_{0}$ for the iterations is selected to be the solution to the homogeneous equation $L[y]=0$ subject to (18). Due to the lack of proper justification on the choice of the sequence $\alpha_{n}$, it is approximated by trying arbitrary values close to one and compare the results to find the value that meets a certain specified tolerance.

\subsection{The Iterative Scheme for the Solution of Second Order ODEs}

Consider the following generalized second order ODE where $L$ is a linear differential operator representing the second order derivative and $f(x)$ is the inhomogeneous term.

$$
L[y] \equiv y^{\prime \prime}(x)+q(x) y^{\prime}(x)+r(x) y(x)=f(x)
$$

subject to the following boundary conditions:

$$
\begin{aligned}
& B_{a}[y] \equiv a_{0} y(a)+a_{1} y^{\prime}(a)=B_{1} \\
& B_{b}[y] \equiv b_{0} y(b)+b_{1} y^{\prime}(b)=C_{1}
\end{aligned}
$$

The general solution to (32) is given as:

$$
y=y_{h}+y_{p}
$$

In (35), $y_{h}$ is the homogeneous solution which satisfies $L[y]=0$ and the boundary conditions given by (33) and (34). While $y_{p}$ is the particular solution which satisfies $L[y]=f(x)$ with the following boundaries $B_{a}[y]=B_{b}[y]=0$.

The particular solution to (32) is represented as:

$$
y_{p}=\int_{a}^{b} G(x, s) f(s) d s
$$

The Green's function $G(x, s)$ is defined as:

$$
L[G(x, s)]=\delta(x-s)
$$

subject to:

$$
B_{a}[G(x, s)]=B_{b}[G(x, s)]=0
$$


To construct the Green's function, let $y_{1}$ and $y_{2}$ form the fundamental set of solutions for $L[y]=0$, which is the homogeneous solution. For $x \neq s$, the Green's function is a homogeneous solution to (32); however, when $x=s$ the Green's function has singular behavior. Therefore, the Green's function comprises of two identical solutions for each side of the Dirac delta function given as:

$$
G(x, s)= \begin{cases}f_{1} y_{1}+f_{2} y_{2} & a \leq x<s \\ g_{1} y_{1}+g_{2} y_{2} & s<x \leq b\end{cases}
$$

where $f_{1}, f_{2}, g_{1}$ and $g_{2}$ are constants; these constants are to be found using the following properties:

$G(x, s)$ satisfies the homogeneous boundary conditions:

$$
B_{a}[G(x, s)]=B_{b}[G(x, s)]=0
$$

Continuity of $G(x, s)$ at $x=s$ :

$$
f_{1} y_{1}(s)+f_{2} y_{2}(s)=g_{1} y_{1}(s)+g_{2} y_{2}(s)
$$

Jump discontinuity of $G(x, s)$ at $x=s$ :

$$
g_{1} y_{1}{ }^{\prime}(s)+g_{2} y_{2}{ }^{\prime}(s)-f_{1} y_{1}{ }^{\prime}(s)-f_{2} y_{2}{ }^{\prime}(s)=1
$$

For the implementation of the MGEM, we first define the following linear integral operator:

$$
K[y]=y_{h}+\int_{a}^{b} G(x, s)\left[y^{\prime \prime}(s)+q(s) y^{\prime}(s)+r(s) y(s)\right] d s
$$

Now adding and subtracting $f(x)$ from (43), we get:

$$
\begin{aligned}
K[y]= & y_{h}+\int_{a}^{b} G(x, s)\left[y^{\prime \prime}(s)+q(s) y^{\prime}(s)+r(s) y(s)-f(s)\right] \\
& d s+\int_{a}^{b} G(x, s) f(s) d s
\end{aligned}
$$

Using (35) and (36) in (44), we obtain:

$$
\begin{aligned}
K[y]= & y_{h}+\int_{a}^{b} G(x, s)\left[y^{\prime \prime}(s)+q(s) y^{\prime}(s)+r(s) y(s)-f(s)\right] \\
& d s+y-y_{h}
\end{aligned}
$$

Therefore, from (45), we find:

$$
K[y]=y+\int_{a}^{b} G(x, s)\left[y^{\prime \prime}(s)+q(s) y^{\prime}(s)+r(s) y(s)-f(s)\right] d s
$$

Mann's fixed point iterative scheme is known as:

$$
y_{n+1}=\left(1-\alpha_{n}\right) y_{n}+\alpha_{n} K[y] \quad \text { for } \quad n \geq 0
$$

Applying (47) to (46) yields:

$$
y_{n+1}=y_{n}+\alpha_{n} \int_{a}^{b} G(x, s)\left[y_{n}{ }^{\prime \prime}(s)+q(s) y_{n}^{\prime}(s)+r(s) y_{n}(s)-f(s)\right] d s
$$

For $n=0$, the starting function $y_{0}$ for the iterations is selected to be the solution to the homogeneous equation $L[y]=0$ subject to (33) and (34). The choice of the sequence $\alpha_{n}$ is similar to section 3.1.

\subsection{Construction of the Iterative Scheme for the Biofilter Model Equations}

In this section, the MGEM developed in section 3 is applied to the BF model equations shown by (9), (10), (13) and (14).

The iterative schemes for the biofilm phase model equations are:

$$
\begin{aligned}
S_{m(n+1)}^{*}= & S_{m(n)}^{*}+\alpha_{n}\left[\int_{0}^{\sigma}(-s)\left(\frac{d^{2} S_{m(n)}^{*}}{d \sigma^{2}}-\frac{\phi_{1} S_{m(n)}^{*}}{1+\beta_{1} C_{m(n)}^{*} S_{m(n)}^{*}}\right) d s+\right. \\
& \left.+\int_{\sigma}^{1}(-\sigma)\left(\frac{d^{2} S_{m(n)}^{*}}{d \sigma^{2}}-\frac{\phi_{1} S_{m(n)}^{*}}{1+\beta_{1} C_{m(n)}^{*} S_{m(n)}^{*}}\right) d s\right]
\end{aligned}
$$

$$
\begin{aligned}
S_{p(n+1)}^{*}= & S_{p(n)}^{*}+\alpha_{n}\left[\int_{0}^{\sigma}(-s)\left(\frac{d^{2} S_{p(n)}^{*}}{d \sigma^{2}}-\frac{\alpha \phi_{2} S_{p(n)}^{*}}{1+\beta_{2} C_{p(n)}^{*} S_{p(n)}^{*}}\right) d s+\right. \\
& \left.\int_{\sigma}^{1}(-\sigma)\left(\frac{d^{2} S_{p(n)}^{*}}{d \sigma^{2}}-\frac{\alpha \phi_{2} S_{p(n)}^{*}}{1+\beta_{2} C_{p(n)}^{*} S_{p(n)}^{*}}\right) d s\right]
\end{aligned}
$$

where the starting points for the iterations are $S_{m(n=0)}^{*}=1$ and $S_{p(n=0)}^{*}=1$.

The iterative schemes for the gas phase model equations are:

$$
\begin{aligned}
C_{m(n+1)}^{*} & =C_{m(n)}^{*}+\alpha_{n}\left[\int_{0}^{\xi}(-1)\left(\frac{d C_{m(n)}^{*}}{d \xi}-\kappa_{1} C_{m(n)}^{*}\left(\frac{d S_{m}^{*}}{d \sigma}\right)_{\sigma=0}\right) d s\right] \\
C_{p(n+1)}^{*} & =C_{p(n)}^{*}+\alpha_{n}\left[\int_{0}^{\xi}(-1)\left(\frac{d C_{p(n)}^{*}}{d \xi}-\kappa_{2} C_{p(n)}^{*}\left(\frac{d S_{p}^{*}}{d \sigma}\right)_{\sigma=0}\right) d s\right]
\end{aligned}
$$

where the starting points for the iterations are $C_{m(n=0)}^{*}=1$ and $C_{p(n=0)}^{*}=1$

\section{Simulation Results and Discussion}

\subsection{Biodegradation of Methanol and Alpha-pinene}

In order to verify numerically whether MGEM leads to accurate solutions, the iterative schemes developed in section 4 (Eq. 49-53) were applied to reproduce the concentration profiles of $\mathrm{MeOH}$ and alpha-pinene published in Mohseni and Allen [19]. It is worth mentioning that all the required simulations were carried out utiliz- 
Table 1. Parameter Values [19].

\begin{tabular}{lccccc}
\hline Parameter & Value & Unit & Parameter & Value & Unit \\
\hline$A_{s}$ & 70.0 & $\mathrm{~m}^{2} \cdot \mathrm{m}^{-3}$ & $r_{m}^{*}$ & 98,000 & $\mathrm{~g} \cdot \mathrm{m}^{-3} \mathrm{~h}^{-1}$ \\
$H$ & 1.00 & $\mathrm{~m}$ & $r_{p}^{*}$ & 18,000 & $\mathrm{~g} \cdot \mathrm{m}^{-3} \mathrm{~h}^{-1}$ \\
$\delta$ & $1.00 \times 10^{-4}$ & $\mathrm{~m}$ & $K_{s(m)}$ & 95.2 & $\mathrm{~g} \cdot \mathrm{m}^{-3}$ \\
$D_{m}$ & $3.40 \times 10^{-6}$ & $\mathrm{~m}^{2} \cdot \mathrm{h}^{-1}$ & $K_{s(p)}$ & 8.20 & $\mathrm{~g} \cdot \mathrm{m}^{-3}$ \\
$D_{p}$ & $1.20 \times 10^{-6}$ & $\mathrm{~m}^{2} \cdot \mathrm{h}^{-1}$ & $K_{I}$ & 0.23 & $\mathrm{~g} \cdot \mathrm{m}^{-3}$ \\
$m_{m}$ & 0.01 & - & $C_{i(m)}$ & 1.00 & $\mathrm{~g} \cdot \mathrm{m}^{-3}$ \\
$m_{p}$ & 0.011 & - & $C_{i(p)}$ & 0.26 & $\mathrm{~g} \cdot \mathrm{m}^{-3}$ \\
\hline
\end{tabular}

* is the growth rate constant defined as $r=\frac{X_{v} \mu_{\max }}{Y}$.

ing Computer Algebra System MAPLE 17 software. The numerical predictions were compared with both the experimental data and the originally published model predictions presented in [19]. The parameter values used for solving the model equations are given in Table 1.

\subsection{Biofiltration of Methanol}

For the biofiltration of $\mathrm{MeOH}$, a tolerance of $10^{-4}$ is imposed on the residual error as a stopping criterion for the iterations. Several simulations runs have been done with a different number of iterations and various sequence $\alpha_{n}$ values. The corresponding maximum residual errors were estimated along the $\mathrm{BF}$ height to determine the number of iterations and the proper sequence setting that yields to the specified tolerance. The maximum residual errors for 15, 20 and 25 iterations at $\alpha_{n}$ equal to $0.6,0.4$ and 0.2 are reported in Tables S1 to S3. The results showed that at a given value of $\alpha_{n}$, the maximum residual error decreases with increasing number of iterations. To plot the concentration profile of $\mathrm{MeOH}, 15$ iterations and a sequence of 0.4 were used as they meet the specified stopping criteria as mentioned above. Fig. 2 shows that the proposed technique yields a highly accurate solution and it can describe the biofiltration of $\mathrm{MeOH}$ when compared against the original model solution and the experimental data from the literature.

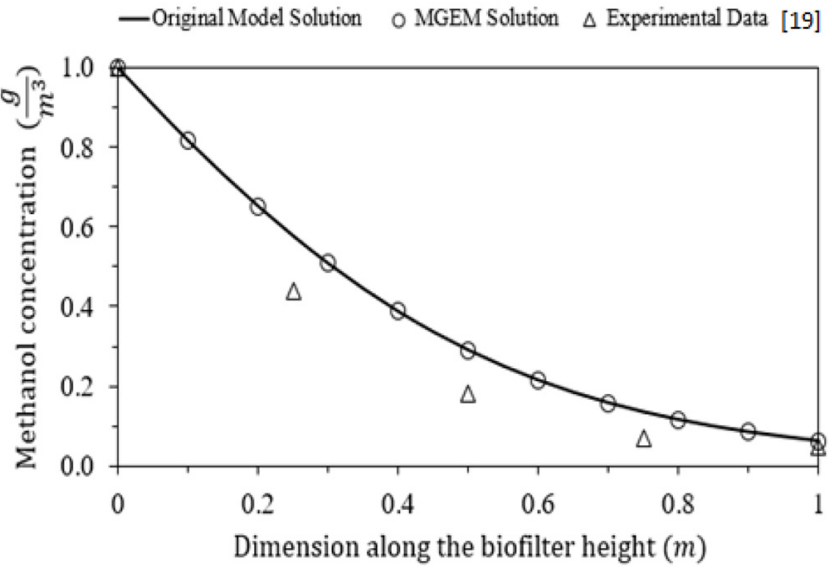

Fig. 2. Methanol concentration profile along the biofilter height.

\subsection{Biofiltration of Alpha-pinene}

For the biofiltration of alpha-pinene (Fig. 3), the numerical results showed that Mann's Green's embedded method (MGEM) permitted a detailed description of alpha-pinene biofiltration when 50 iterations and a sequence of 0.18 were used. The optimal number of iterations and the proper setting for $\alpha_{n}$ were determined by carrying out several simulations runs to meet a tolerance of $10^{-4}$. These simulations were also done to study the impact of the number of iterations and the sequence value on the residual error. The residual error results are shown for 50,55 and 60 iterations at $\alpha_{n}$ equal to 0.2 , 0.18 and 0.1 in Tables S4 to S6. The results showed that the residual error improves when more iteration are carried out.

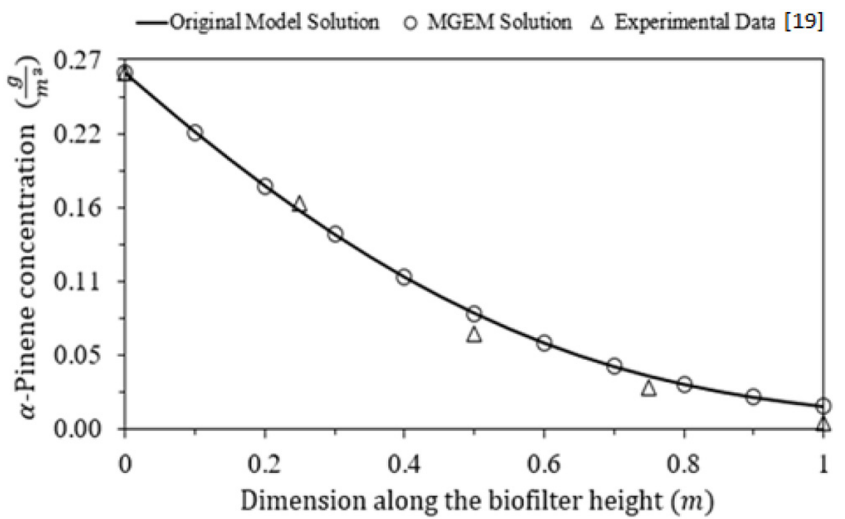

Fig. 3. Alpha-pinene concentration profile along the biofilter height.

\subsection{Sensitivity Analysis}

One of the main objectives in the mathematical modeling of BFs is to analyze the impact of different parameters on the biofiltration process. This may help to have a clear understanding of the physical, chemical and biological phenomena. In this section, the MGEM with 15 iterations and a sequence of 0.4 was utilized to perform a sensitivity analysis on the model equations. The analysis performed here focused on studying the effect of empty bed residence time (EBRT), biofilm surface area, diffusion coefficient and air-biofilm partition coefficient on the $\mathrm{RE}$ of $\mathrm{MeOH}$. The analysis was done by varying one parameter from Table 1 while maintaining the other parameters fixed at constant values. 


\subsection{Effect of Empty Bed Residence Time of the RE}

Fig. 4 shows the $\mathrm{BF}$ performance based on the $\mathrm{RE}$ of $\mathrm{MeOH}$ under different EBRT (5-130 s) at an inlet $\mathrm{MeOH}$ concentration of 1 g.m ${ }^{-3}$. $\mathrm{RE}$ increased rapidly when the EBRT was varied from $5 \mathrm{~s}$ to around $30 \mathrm{~s}$. For short EBRT of around $10 \mathrm{~s}$ and less, the BF exhibited low $\mathrm{RE}$ of $\mathrm{MeOH}(\leq 60 \%)$. This is attributed to the fact that, the contact between the biomass and $\mathrm{MeOH}$ was too quick at which the microorganisms had insufficient time to perform the required degradation on the available amount of $\mathrm{MeOH}$. In contrast, for long EBRT the RE increased until it reached a complete elimination of $\mathrm{MeOH}$ at an EBRT of $50 \mathrm{~s}$. The increase in the RE can be explained by the fact that the time spent by $\mathrm{MeOH}$ in the $\mathrm{BF}$ is sufficiently large for the process to effectively remove the pollutant.

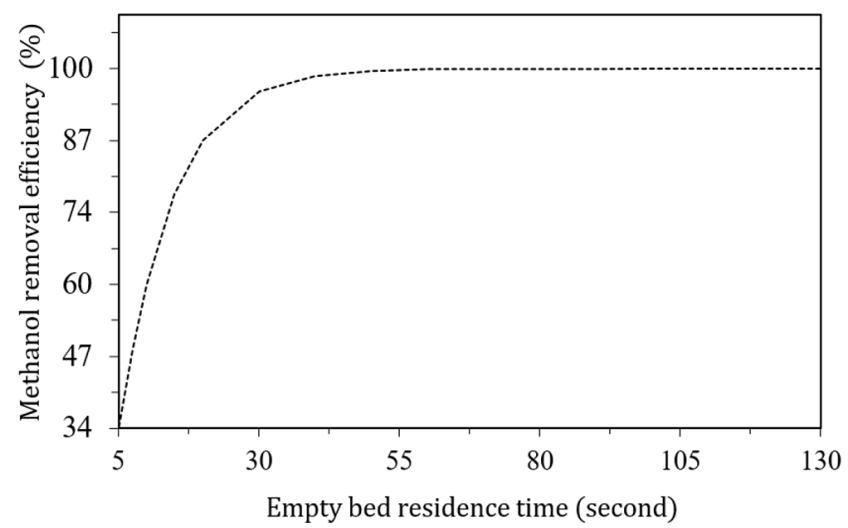

Fig. 4. Removal efficiency of methanol vs. the empty bed residence time.

\subsection{Effect of Biofilm Surface Area on the RE}

The $\mathrm{RE}$ of $\mathrm{MeOH}$ is plotted as a function of the biofilm surface area at an inlet $\mathrm{MeOH}$ concentration of $1 \mathrm{~g} \cdot \mathrm{m}^{-3}$ and an EBRT of $50 \mathrm{~s}$ in Fig. 5. The results showed that the RE is an increasing function of the biofilm surface area. The analysis demonstrated that a complete removal of $\mathrm{MeOH}$ can be accomplished at a biofilm surface area of $63 \mathrm{~m}^{2} \cdot \mathrm{m}^{-3}$. This is intuitively expected because for a given biofilm thickness increased surface area increases the reaction volume and area for mass transfer.

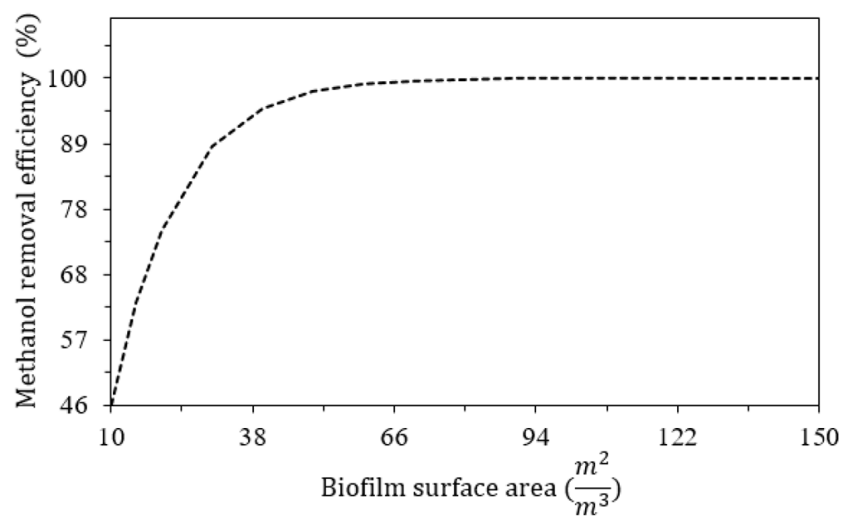

Fig. 5. Removal efficiency of methanol vs. the biofilm surface area.

\subsection{Effect of Air-biofilm Partition Coefficient on the RE}

Fig. 6 shows the $\mathrm{RE}$ of $\mathrm{MeOH}$ as a function of the air-biofilm partition coefficient at an inlet $\mathrm{MeOH}$ concentration of 1 g.m $\mathrm{m}^{-3}$ and an EBRT of $50 \mathrm{~s}$. Although the air-biofilm partition coefficient is constant for a given compound, this parameter could be altered by factors such as temperature and surfactant addition. Therefore, it is essential to examine the effect of this parameter on the RE. At low values of air-biofilm partition coefficient of up to 0.01 , the BF showed $100 \%$ removal of $\mathrm{MeOH}$. This phenomenon is due to the fact that at low values of the air-biofilm partition coefficient, the solubility of $\mathrm{MeOH}$ in the biofilm increases resulting in rapid biodegradation in the biofilm. However, the increase in the air-biofilm partition coefficient to 0.1 substantially reduced the RE to $40.87 \%$. This analysis confirms that the $\mathrm{RE}$ of the biological system diminishes as the air-biofilm partition coefficient increases since this hinders the diffusion of pollutants into the biofilm where the elimination process occurs.

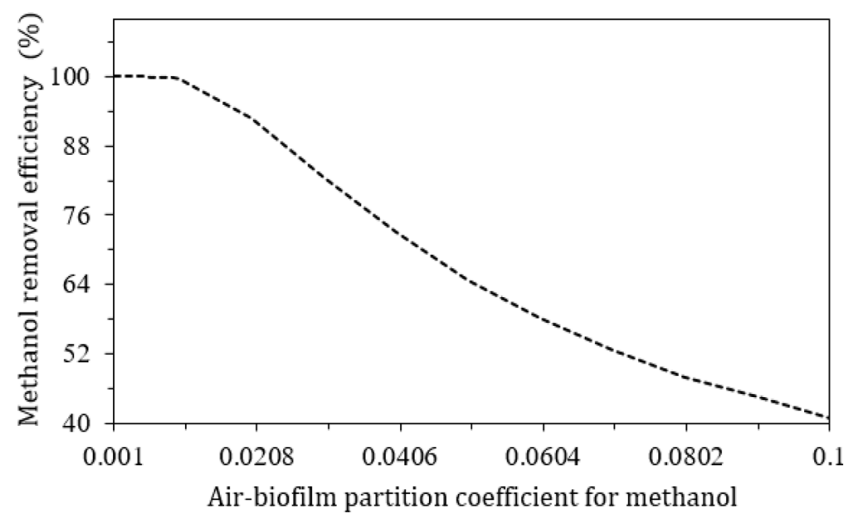

Fig. 6. Removal efficiency of methanol vs. the air-biofilm partition coefficient.

\subsection{Effect of Diffusion Coefficient on the RE}

The diffusion coefficient describes the rate at which a pollutant is transferred from the gas phase to the biofilm phase. This parameter significantly impacts the RE of pollutants in BFs as it depends upon the biofilm density and the operating temperature. Consequently, the effect of this parameter on the $\mathrm{RE}$ of $\mathrm{MeOH}$ is analyzed in Fig. 7. The diffusion coefficient was varied in

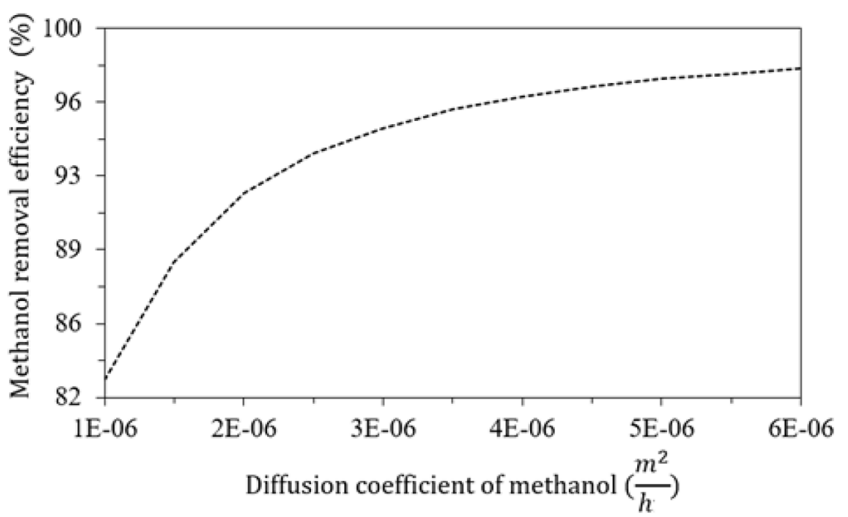

Fig. 7. Removal efficiency of methanol vs. the diffusion coefficient. 
crementally from low values of about $1.00 \times 10^{-6} \mathrm{~m}^{2} \cdot \mathrm{h}^{-1}$ to 6.00 $\times 10^{-6} \mathrm{~m}^{2} \cdot \mathrm{h}^{-1}$ at an inlet MeOH concentration of $1 \mathrm{~g} \cdot \mathrm{m}^{-3}$ and EBRT of $50 \mathrm{~s}$. The simulation results show that the RE increases as the diffusion coefficient is increased. This behavior is expected as at high values of the diffusion coefficient, $\mathrm{MeOH}$ diffuses faster into the biofilm where the biodegradation takes place. A maximum $\mathrm{RE}$ of $98.06 \%$ was noticed at a diffusion coefficient of $6.00 \times$ $10^{-6} \mathrm{~m}^{2} \cdot \mathrm{h}^{-1}$. While at low diffusivity of $1.00 \times 10^{-6} \mathrm{~m}^{2} \cdot \mathrm{h}^{-1}$ the $\mathrm{BF}$ removed about $82.83 \%$ of the $\mathrm{MeOH}$.

\section{Conclusion}

In this work, a novel technique known as Mann's Green's embedded method (MGEM) is successfully applied to obtain an approximate solution for a model which describes biofiltration of methanol and alpha-pinene, which are key hazardous air pollutants emitted from the pulp and paper industry [19]. The results obtained for each compound were compared against experimental data from the literature and the original model predicted solution. The Mann's Green's embedded method (MGEM) is able to provide excellent numerical approximations for the biofiltration of both compounds. Furthermore, with this MGEM based model, a sensitivity analysis of important parameters (empty bed residence time, biofilm surface area, diffusion coefficient and air-biofilm partition coefficient) on the removal efficiency (RE) of $\mathrm{MeOH}$ was performed to analyze the influence of these parameters on the biofiltration process. The model predictions show that these parameters highly influence removal performance; and thus, accurate estimations of these parameters are important. It should be mentioned that kinetics of biodegradation for these two compounds, methanol and alpha-pinene, are non-linear in nature as described by Monod kinetics. MGEM has been established in solving nonlinear boundary value problems with high accuracy as compared to exact and existing numerical solutions without altering physical representation of the problem (i.e. without changing the boundary conditions). Furthermore, this scheme converges fast and can be applied to a wide spectrum of problems in the physical sciences and engineering [30]. To the authors' knowledge, this is the first time Mann's Green's embedded method (MGEM) is applied in the solution of biofilter models. Most biological reactor equations yield boundary value problems with highly non-linear kinetics. Therefore, the method presented and outcomes of this contribution are expected to be valuable for scientific community who work in the area of bioreactor modeling.

\section{Author Contributions}

M.S. (M.Sc. Student) did the simulation and worked on the draft manuscript. Z.S. (Professor) advised the project throughout and revised the manuscript. S.K. (Professor) helped in the solution of the mathematical models.

\section{References}

1. Kumar TP, Rahul, Kumar MA, Chandrajit B. Biofiltration of volatile organic compounds (VOCs)-An overview. Res. J. Chem. Sci. 2011:1:83-92.

2. Meunier F. The greenhouse effect: A new source of energy. Appl. Therm. Eng. 2007:27:2-3:658-664.

3. Shareefdeen ZM, Singh A. Biotechnology for odor and air pollution control. Berlin: Springer-Verlag: 2005.

4. Kennes C, Veiga MC. Bioreactors for waste gas treatment. Netherlands: Springer; 2001.

5. Mudliar S, Giri B, Padoley K, et al. Bioreactors for treatment of VOCs and odours-A review. J. Environ. Manage. 2010:91: 1039-1054

6. Malakar S, Saha PD, Baskaran D, Rajamanickam R. Comparative study of biofiltration process for treatment of VOCs emission from petroleum refinery wastewater-A review. Environ. Technol. Innov. 2017:8:441-461.

7. Gribbins M), Loehr RC. Effect of media nitrogen concentration on biofilter performance. J. Air Waste Manage. Assoc. 1998:48: 216-226.

8. Morgenroth E, Schroeder ED, Chang YDP, Scow KM. Nutrient limitation in a compost biofilter degrading hexane. J. Air Waste Manag. Assoc. 1996:46:300-308.

9. Sagastume FM, Sleep BE, Allen DG. Effects of biomass growth on gas pressure drop in biofilters, J. Environ. Eng. 2001:127: 388-396.

10. Smet E, Chasaya G, Langenhove HV, Verstraete W. The effect of inoculation and the type of carrier material used on the biofiltration of methyl sulphides. Appl. Microbiol. Biotechnol. 1996:45:293-298.

11. Smet E, Langenhove HV, Philips G. Dolomite limits acidification of a biofilter degrading dimethyl sulphide. Biodegradation 1999:10:399-404.

12. Yang H, Minuth B, Allen DG. Effects of oxygen and nitrogen on biofilter performance. J. Air Waste Manage. Assoc. 2002:52 279-286.

13. Alvarez-Hornos FJ, Gabadlón C, Martínez-Soria V, Marzal P, Penya-Roja JM. Mathematical modeling of the biofiltration of ethyl acetate and toluene and their mixtures. Biochem. Eng. J. 2009:43:169-177

14. Baquerizo G, Maestre JP, Sakuma T, et al. A detailed model of a biofilter for ammonia removal: Model parameters analysis and model validation. Chem. Eng. J. 2005:113:205-214.

15. De Visscher D, Van Cleemput O. Simulation model for gas diffusion and methane oxidation in landfill cover soils. Waste Manage. 2003:23:581-591.

16. Deshusses MA, Hamer G, Dunn IJ. Behavior of biofilters for waste air biotreatment.1. dynamic model development. Environ. Sci. Technol. 1995:29:1048-1058.

17. Dorado AD, Lafuente J, Gabriel D, Gamisans X. Biomass accumulation in a biofilter treating toluene at high loads- part 2: Model development, calibration and validation. Chem. Eng. J. 2012:209:670-676.

18. Hodge DS, Devinny JS. Modeling removal of air contaminants by biofiltration, J. Environ. Eng. 1995:121:21-32.

19. Mohseni M, Allen DG. Biofiltration of mixtures of hydrophilic and hydrophobic volatile organic compounds. Chem. Eng. Sci. 2000:55:1545-1558.

20. Nikiema J, Payre G, Heitz M. A mathematical steady state model 
for methane bioelimination in a closed biofilter. Biochem. Eng. J. 2009:150:418-425.

21. Ottengraf SPP, Van Den Oever AHC. Kinetics of organic compound removal from waste gases with a biological filter. Biotechnol. Bioeng. 1983:25:3089-310.

22. Ottengraf SPP. Exhaust gas purification. Biotechnol. Bioeng. 1986:8:425-452.

23. Rahul, Mathur AK, Bala S, Majumder C. Modelling and computational fluid dynamic behaviour of a biofilter treating benzene. Bioresour. Technol. 2012:125:200-207.

24. Shareefdeen ZM, Baltzis BC, Oh YS Bartha R. Biofiltration of methanol vapor. Biotechnol. Bioeng. 1993:41;512-524.

25. Shareefdeen ZM, Baltzis BC. Biofiltration of toluene vapor under steady state and transient conditions: theory and experimental results. Chem. Eng. Sci. 1994:49:4347-4360.

26. Zhang Y, Liss SN, Allen DG. Enhancing and modeling the biofiltration of dimethyl sulfide under dynamic methanol addition. Chem. Eng. Sci. 2007:62:2474-2481.

27. Leson G, Winer AM. Biofiltration: an innovative air pollution control technology for VOC emissions. J. Air Waste Manage. Assoc. 1991:41:1045-1054.

28. Abushammala M, Khuri SA, Sayfy A. A novel fixed point iteration for the solution of third order boundary value problems. Appl. Math. Comput. 2015:182;536-543.

29. Berinde V. Iterative approximation of fixed points. Lecture Notes in Mathematics. Berlin; Springer: 2007.

30. Dehaish BAB, Khamsi MA. Mann iteration process for monotone non-expansive mappings. Fix. Point Theory Appl. 2015:177. 